
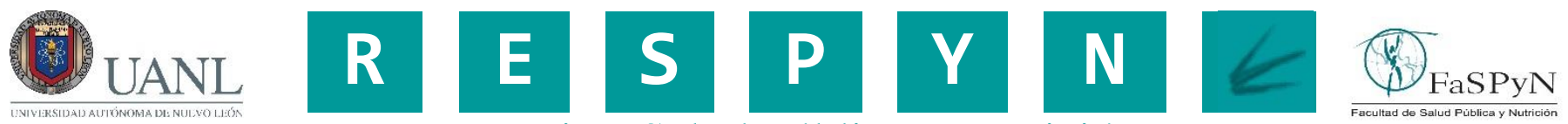

Revista Salud Pública y Nutrición

\title{
EFECTO DE SIETE DIETAS POPULARES EN EL PESO Y LA COMPOSICIÓN CORPORAL EN ADULTOS: UNA REVISIÓN SISTEMÁTICA.
}

\author{
EFFECT OF SEVEN POPULAR DIETS ON WEIGHT AND BODY COMPOSITION IN ADULTS: A SYSTEMATIC \\ REVIEW.
}

Winterman Hemilson Bertha*, Ramírez López Erik*, Medellín Guerrero Alpha Berenice*.

* Universidad Autónoma de Nuevo León, México.

Citation: Winterman Hemilson B., Ramírez López E., Medellín Guerrero A.B. (2021) Efecto de siete dietas populares en el peso y la composición corporal en adultos: una revisión sistemática. Revista Salud Pública y Nutrición, 20 (1), 30-39.

Editor: Esteban G. Ramos Peña, Dr. CS., Universidad Autónoma de Nuevo León, Facultad de Salud Pública y Nutrición, Monterrey Nuevo León, México. Copyright: @2021 Winterman Hemilson B., et al. This is an open-access article distributed under the terms of Creative Commons Attribution License [CC BY 4.0], which permits unrestricted use, distribution, and reproduction in any medium, provided the original author and source are credited.

Competing interests: The authors have declared that no competing interests exist.

DOI: https://doi.org/10.29105/respyn20.1-4

Recibido: 18 de octubre 2020;

Aceptado: 11 de diciembre 2020

Email: berthita3@gmail.com 


\title{
EFECTO DE SIETE DIETAS POPULARES EN EL PESO Y LA COMPOSICIÓN CORPORAL EN ADULTOS: UNA REVISIÓN SISTEMÁTICA.
}

\author{
Winterman Hemilson Bertha*, Ramírez López Erik*, Medellín Guerrero Alpha Berenice*. \\ * Universidad Autónoma de Nuevo León, México.
}

\section{RESUMEN}

Introducción. Si una dieta ofrece reducir de peso en un periodo corto, se le define como dieta de moda o milagro. No es claro su efecto en el peso y la composición corporal (CC). Objetivo: resumir los antecedentes y sintetizar los efectos de siete dietas populares en el peso y la CC en adultos. Métodos: Se usaron cinco bases de datos entre las cuales están PubMed y Cochcrane. Los términos usados en la búsqueda fueron dieta de moda, dieta milagro, dieta popular, CC, masa grasa, músculo, entre otros. Se combinaron con operadores lógicos y términos como dieta Atkins, dieta Dukan, dieta de la Zona, Ayuno Intermitente, dieta Paleolítica, dieta Vegetariana, Vegana, adultos entre otros. Resultados: Se identificaron ocho metanálisis que contenían ensayos clínicos aleatorizados y se consideraron para la revisión seis. Otros 43 ensayos fueron consultados para obtener detalles. Los metaanálisis excluidos no cumplieron con criterios de los niveles de evidencia y de la guía PRISMA. Conclusiones: Las dietas populares analizadas podrían ocasionar perdidas de peso en el corto y largo plazo de entre 4.3 y $10 \mathrm{~kg}$. Los cambios en la composición corporal no han sido satisfactoriamente reportados. Aun es limitada la información para generalizar los cambios en el peso y la CC por efecto de las dietas populares.

Palabras Clave: Dieta popular, composición corporal, peso corporal.

\section{ABSTRACT}

Introduction: If a diet offers to reduce weight in a short period, it is defined as a fad or miracle diet. The effect of popular diets in the short or long term on weight and body composition is not exact. Objective: To summarize the background and synthesize the effects of seven popular diets on weight and body composition in adults. Methods: Five databases were used, including PubMed and Cochrane. The terms used in the search were fad diet, miracle diet, popular diet, CC, fat mass, and muscle mass, among other terms. They were combined with logical operators and terms such as Atkins diet, Dukan diet, Zone diet, Intermittent Fasting, Paleolithic diet, Vegetarian diet, Vegan, and adults. Results: Eight meta-analyses containing randomized clinical trials were identified, and six were considered for review. Another 43 trials were consulted for details. The excluded meta-analyses did not meet the criteria for levels of evidence and the PRISMA guideline. Conclusions: the popular diets analyzed could cause weight loss in the short and long term between 4.3 and $10 \mathrm{~kg}$. Changes in body composition have not been satisfactorily reported. Information is still limited to generalize changes in weight and WC due to popular diets.

Key words: Popular diet, corporal composition, body weight. 


\section{Introducción}

Las personas suelen elegir ciertas dietas para disminuir de peso o bien para modificar sus hábitos o estilo de vida. Si una dieta ofrece reducir de peso en un periodo corto, menor de 6 meses, se le define como dieta de moda o milagro. Estos términos no aplicarían a dietas como la vegetariana o paleolítica, que buscan modificar el estilo de vida más que reducir el peso de forma acelerada. Para fines prácticos, nos referiremos a las dietas milagro o de moda y a las de cambio en el estilo de vida como dietas populares. En la práctica nutricional, se suele asumir que una dieta popular podría ocasionar cambios rápidos o desfavorables en el peso y la composición corporal de un paciente. Sin embargo, no es claro el efecto de estas dietas en el corto o largo plazo en el peso, la grasa corporal, masa muscular y otros componentes. El objetivo de esta revisión es resumir los antecedentes y sintetizar los efectos de siete dietas populares en el peso y la composición corporal en adultos.

\section{Metodología}

Esta es una revisión sistemática cualitativa descriptiva (sin análisis estadístico). Se incluyeron estudios en centros clínicos, laboratorios de investigación y población abierta. El intervalo de edad seleccionado fue de 18 a 75 años. Se incluyeron estudios con pacientes con diabetes, hipertensión u otras enfermedades crónicas además de sujetos sanos con sobrepeso u obesidad. Se incluyeron los ensayos que informaron la aplicación de las dietas modificándolas o agregando actividad física cuando ningún otro estudio pudo reemplazarlos. Para resolver el problema o tema de revisión se elaboró la pregunta PICO considerando los siguientes elementos: P; paciente o problema: estudios con pacientes que emplean dietas para bajar de peso o modificar su estilo de vida. I; intervención: dietas milagro o de moda modificadas en la proporción de macronutrientes y/o reducidas en calorías. C; Comparación dieta normal, habitual u otra dieta de moda. O; outcome o resultado: donde se muestren resultados en el peso, masa grasa, masa magra, músculo, grasa regional o visceral, agua corporal, masa o densidad ósea y peso. Por lo tanto, la pregunta PICO fue la siguiente: en las personas que usan dietas para bajar de peso o modificar su estilo de vida ¿cuáles son los efectos de la implementación de las dietas populares sobre el peso y la composición corporal? Para contestar esta pregunta, el presente estudio se ajustó a la declaración PRISMA para revisiones sistemáticas y metanálisis. Se realizó una búsqueda en idioma inglés y español en las bases de datos Pubmed, Google Scholar, Cochrane, Latindex, LILACS y Scielo. Se comprobó que los artículos no estuvieran en la lista de journals depredadores (List of Predatory Journals). Si los artículos eran de acceso libre, se confirmó su inclusión en la base Directory of Open Access Journals (DJOA). Se emplearon términos generales en castellano y en inglés como dieta de moda, dieta milagro, dieta popular, composición corporal, masa magra, masa libre de grasa, músculo, grasa visceral, agua corporal, grasa regional, porcentaje de grasa, masa ósea, densidad ósea, dieta Atkins, dieta Dukan, dieta de la zona, ayuno intermitente, dieta paleolítica, dieta vegetariana y dieta vegana. Otros términos empleados y combinados con los operadores booleanos (NOT, OR y AND) fueron sobrepeso, obesidad, diabetes y adulto. La búsqueda se concretó en los últimos diez años y se seleccionaron metaanálisis de estudios aleatorios controlados que tuvieran el análisis de GRADE u otra fuente de evaluación de nivel de calidad basada en evidencia. Los metaanálisis fueron revisados individualmente y analizados para obtener los datos puntuales de cambio en el peso y la composición corporal por grupo o fase de intervención.

\section{Desarrollo y discusión}

Dieta normal y pérdida de peso de acuerdo con las normas. Una dieta normal puede definirse como el conjunto de alimentos y platillos que se consumen cada día y constituye la unidad de la alimentación (NOM-043, 2012 "Promoción y educación para la salud en materia alimentaria. Criterios para brindar orientación"). Existen dietas normales y terapéuticas; dentro de estas últimas están las dietas para el control de peso y tratamiento de la obesidad (Pérez- Lizaur y García-Campos, 2012). Por otro lado, están las dietas populares, donde algunas tienen como objetivo promover la pérdida de peso y otras como las vegetarianas, fomentar un estilo de vida en particular (Centurión-Bernal, González-Acosta, Rojas-Pavón, Burgos-Larroza \& Meza-Miranda, 2018). El Centro de Investigación para la diabetes Joslin de EUA (Hamdy, 2011) sugiere que perder entre 5 a 10\% del peso inicial mejora de forma significativa el control de la glucosa en sangre en pacientes con diabetes y ayuda en la prevención de la prediabetes. En México, se sugiere que los pacientes con obesidad (IMC > 30 
$\mathrm{kg} / \mathrm{m} 2$ ) podrán tener una restricción energética de 20 a $25 \mathrm{kcal} /$ día con base en el "peso ideal". (NOM-015SSA2-2010 "Para la prevención, tratamiento y control de la diabetes mellitus"). De inicio, se sugiere reducir del consumo habitual 250 a $500 \mathrm{kcal} /$ día, hasta alcanzar mínimo $1200 \mathrm{kcal} /$ día. Esto podría lograr una reducción de peso de 0.5 a $1.0 \mathrm{~kg} / \mathrm{semana}$ o $5 \%$ de sobrepeso con mejorías metabólicas. Como es de notar, las guías normas no establecen metas en la modificación de la composición corporal como la grasa o músculo. Esto se debe en parte al acceso de métodos confiables de medición de la composición corporal.

Definición de dieta de moda o milagro. La Federación Mexicana de Diabetes señala que una dieta milagro promete resultados de manera rápida, sin esfuerzo y ocasiona confusión y dudas (FMD, 2014). La Asociación Española de Dietistas Nutricionistas propone identificar a las dietas fraudulentas si prometen resultados rápidos y mágicos; prohíben el consumo de un alimento o grupo, contiene listas de alimentos "buenos", o "malos"; se le atribuyen propiedades extraordinarias a algún alimento, y si en su mayoría incluyen testimonios o relatos de aquellos voluntarios que las hicieron (GREP-AEDN, 2009). La tabla 1 resume las características mencionadas que propone el Grupo de Revisión, Estudio y Posicionamiento de la Asociación Española de Dietistas-Nutricionistas, 2012. Se añaden las sugerencias de la Academia de Nutrición y Dietética para ayudar a los consumidores a detectar alguna dieta de moda (Wolfran, 2019). Para fines prácticos, es este documento nos referiremos a las dietas milagro o de moda y aquellas de cambio en el estilo de vida como dietas populares. Finalmente, una dieta de corto plazo se considera a los 6 meses y una de largo plazo a los 12 meses o más (Antón et al., 2017).

$\underline{\text { Tipos de dietas restringidas en energía o modificadas }}$ en la distribución de macronutrientes. Existe un conocimiento general de cómo podría clasificarse una dieta, pero no un consenso universal con características puntuales. Las tablas 2 y 3 sugieren como podrían clasificarse las distintas dietas populares de acuerdo con su aporte energético, contenido de macronutrientes y elección de ciertos grupos de alimentos.
Efecto de siete dietas populares en la composición corporal. La presente revisión muestra que las dietas populares pueden ser divididas entre dietas de moda (milagro) y aquellas de cambio en el estilo de vida. La etiqueta de dieta milagro depende del uso que se le dé a una dieta en particular. Por ejemplo, está documentado el papel terapéutico de la dieta Atkins en enfermedades como la epilepsia refractaria en niños (Kverneland et al., 2018). Del otro lado, la dieta vegetariana, vegana y paleolítica son consideradas de cambio en el estilo de vida o hábitos. Aunque estas dietas eliminan ciertos alimentos, los principios por los que no aceptan el consumo de estos son diferentes a los que promueven las dietas de moda. A pesar de que se asume que las dietas populares pueden tener efectos adversos o positivos en la composición corporal, la evidencia muestra que estos efectos no han sido reportados de forma suficiente y sistemática. Esto significa que la composición corporal no ha sido el objetivo principal cuando se analiza el efecto de una dieta popular. Por ello, los metaanálisis se centran en el cambio de peso y marcan como limitante de los estudios seleccionados la ausencia de datos de composición corporal. Los cambios en la composición corporal por efecto de una dieta popular deben ser evaluados mediante la pletismografía por desplazamiento de aire (BOD-POD) o la absorciometría dual de rayos $\mathrm{X}$ (DXA) que, no obstante, son costosas y no siempre disponibles en los lugares de investigación. Cuando se usan analizadores de grasa corporal, estos pueden revelar cambios en la composición corporal por efecto de una dieta, pero los resultados entre estudios es difícil compararlos por la variabilidad de las mediciones entre marcas y modelos de dispositivos.

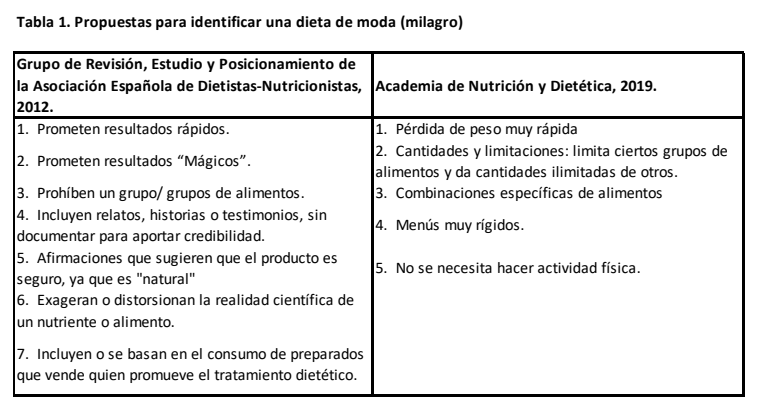

Dieta Atkins y de la Zona (Johnston, et al; 2014). El metaanálisis incluyó estudios con participantes de 46 años ( \pm 9 años), $94.1 \mathrm{~kg}( \pm 14.6 \mathrm{~kg})$ e IMC de 33.7 $\mathrm{kg} / \mathrm{m} 2$ (tabla 4). Con la dieta Atkins se obtuvo una 
pérdida de peso a los 6 meses de $10.1 \mathrm{~kg}$; a los 12 meses de seguimiento, el cambio del peso inicial fue solo de $6.3 \mathrm{~kg}$. Es decir, los pacientes recuperaron 3.8 $\mathrm{kg}$ de peso. Con la dieta de la Zona la pérdida de peso a los 6 meses fue de $8.4 \mathrm{~kg}$, mientras que, a los 12 meses, esta solo fue de $5.9 \mathrm{~kg}$. En este caso la recuperación del peso perdido fue de $2.5 \mathrm{~kg}$. La pérdida de peso a los 6 meses entre ambas dietas solo difirió $1.7 \mathrm{~kg}$ (IC del 95\%, 0,35-3,09 kg). A los 12 meses, la diferencia en la disminución del peso entre las dos dietas solo fue de $0.4 \mathrm{~kg}$. Una limitante importante del estudio de Johnston y colaboradores es que no excluyeron estudios que modificaban la energía de la dieta de acuerdo con las necesidades del paciente $o$ adicionaban actividad física. Estas variables añadidas son un factor de confusión, porque no se está evaluando el efecto único de una dieta sobre el peso y la composición corporal. Por otra parte, la recuperación del peso puede ser debida a la limitada adherencia de los participantes. El porcentaje de abandono de los sujetos en estas dietas fue reportado en promedio en un $40 \%$. Debido a lo anterior, a menudo se cuestiona cual es la mejor dieta, la respuesta podría ser que es aquella a la que el paciente se puede adherir mejor y permanecer tanto tiempo como sea posible.

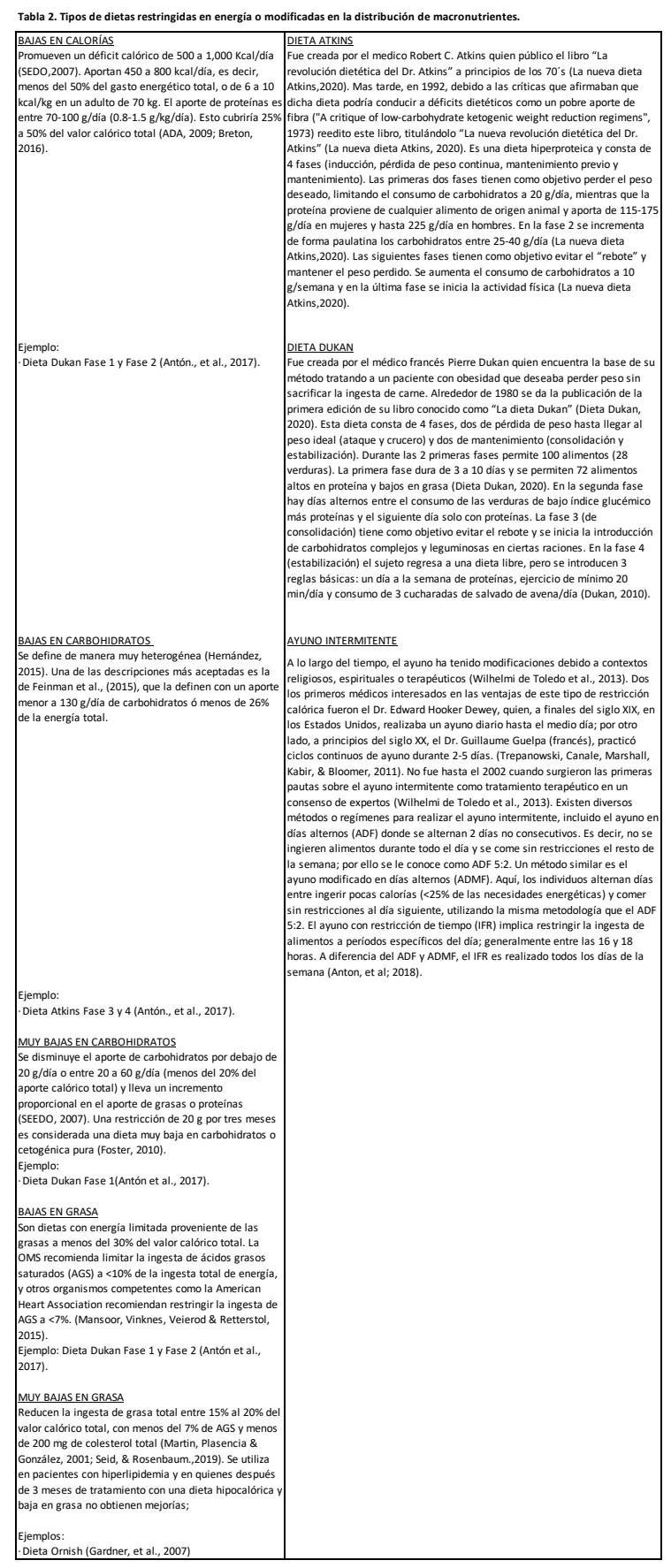

Ayuno intermitente (Cho et al; 2019 y Harris, et al; 2018). Los metanálisis incluyeron participantes que presentaban sobrepeso $\mathrm{u}$ obesidad (IMC $>25 \mathrm{~kg} / \mathrm{m} 2$ ) y edades de 18-65 años (tabla 4). Los estudios incluyeron ensayos que comparan el ayuno intermitente contra una dieta de restricción calórica o 
grupo sin intervención. Entre los diferentes regímenes o métodos de ayuno intermitente el de mayor frecuencia reportado es el ayuno intermitente modificado en días alternos (ver tabla 2). En los días de ayuno se ingiere $<25 \%$ del requerimiento energético total, y el resto de los días se ingiere el $100 \%$ del requerimiento energético. El segundo tipo utilizado fue el ayuno con restricción de tiempo, donde hay una ventana de consumo de energía y otra donde no se ingieren alimentos. Es decir, la ventana de ingesta de energía puede ser diurna o nocturna. Se observó mayor pérdida de peso y mejores parámetros en la resistencia a la insulina en un grupo que consumía más calorías por la mañana y menos por la noche en condiciones de restricción calórica similar. Esto podría repercutir en diferentes cambios de composición corporal. Al analizar los datos, se encontró que a una semana la pérdida de peso es de $1.7 \mathrm{~kg}$ ( $\mathrm{sin}$ especificar si la perdida proviene del componente magro o graso). A un mes la pérdida de peso puede ser hasta de $1.8 \mathrm{~kg}$ del peso basal $(30 \%$ masa grasa y $40 \%$ masa magra); el resto podría deberse a una pérdida de agua por depleción del glucógeno muscular. No obstante, el compartimento de composición corporal más complejo de medir de forma exacta es el agua corporal, por lo que prácticamente no aparece en los ensayos clínicos. Una pérdida de las reservas de glucógeno muscular de 500 g significaría una disminución de 1100 a 1300 $\mathrm{g}$ de agua, por lo que el peso corporal atribuido a esta pérdida seria de $1.8 \mathrm{~kg}$ (Rodoreda, 2013). Esta pérdida de agua no es clínicamente relevante porque solo está en relación con el glucógeno depletado y se recuperaría a medida que el glucógeno se recupera. La pérdida de peso debido al agua puede ser clínicamente relevante cuando a la dieta se añaden diuréticos. A seis meses de intervención el peso total perdido es reportado entre $5.7 \mathrm{~kg}$, de los que $3.6 \mathrm{~kg}$ proviene de la masa grasa (63\%) y $1.2 \mathrm{~kg}$ de la masa magra (21\%). Estos hallazgos se alinean con la sugerencia de que la pérdida de masa magra deseable se mantenga entre $1 / 4$ a $1 / 3$ de la pérdida de peso total en un régimen de restricción calórica continua (Cho et al; 2019).

Dieta paleolítica (Ghaedi et al; 2019 y de Menezes et al., 2019). Se incluyeron sujetos con sobrepeso $u$ obesidad y, además, algunos estudios evaluaron a mujeres post menopaúsicas o personas con diabetes tipo 2 (tabla 4). La pérdida de peso a 1 mes puede ser de hasta $3.4 \mathrm{~kg}$, con una disminución del porcentaje de grasa corporal total de $1.34 \%$. La disminución de peso a los 3 meses puede alcanzar hasta $4.1 \mathrm{~kg}$ del peso inicial. A pesar de no ser una dieta restrictiva en cantidad de alimentos o calorías, el peso perdido a los 6 meses puede ser de hasta $8.1 \mathrm{~kg}$ y $4.3 \%$ de grasa del peso corporal total. Es posible estos cambios se deban en parte a efecto sacietogénico de la dieta, verificado por Bloch et al., 2015, quienes probaron el efecto agudo de las comidas basadas en la dieta paleolítica sobre marcadores bioquímicos de saciedad comparados con otra dieta estándar.

Dieta vegana y vegetariana (Huang, 2015). Se incluyeron sujetos dentro de un rango de edad de 1882 años e IMC inicial entre 25 y $53 \mathrm{~kg} / \mathrm{m} 2$ (tabla 4). La pérdida de peso a un mes en el estilo de vida vegetariano fue de $4.6 \mathrm{~kg}$; a los 3 meses puede ser de $6.5 \mathrm{~kg}$. Es interesante que a los 6 meses la pérdida de peso no se incrementó. La explicación más plausible es que al ser una dieta de cambio en el estilo de vida y no un plan para perder peso, el organismo se adapta a los hábitos modificados y la pérdida de peso se estabiliza. Además, es necesario remarcar que los sujetos generalmente incluidos tienen sobrepeso. En cuánto la dieta vegana, los cambios en la pérdida de peso son similares a los de la dieta vegetariana; a 3 meses de seguimiento, la disminución de peso puede ser de $4.3 \mathrm{~kg}$. A los 6 y 12 meses, la perdida es apenas de 600 gramos (4.8 $\mathrm{kg}$ de peso perdido). El metaanálisis analizado incluye estudios que evalúan el impacto de la dieta junto con una modificación en la dieta o ejercicio. Se descartaron aquellos artículos que incluyeron intervenciones adicionales a la dieta per se, ya que el verdadero efecto de una dieta se mide sin modificación en el aporte de energía y/o actividad física.

Esta revisión solo analizó metaanálisis de los diez últimos años que cumplieran con los criterios PRISMA. Los metanálisis a su vez solo incluyen estudios que cumplen con criterios de calidad del CONSORT y GRADE. Una limitante de los resultados es que el estudio de Johnston, et al; 2014 (Dieta Atkins y de la Zona) no se excluyeron los ensayos que modificaron las dietas de acuerdo con las necesidades de los pacientes o adicionaron actividad física. Por lo tanto, los efectos de las dietas no pueden ser atribuidos a su exclusiva implementación. 


\section{Conclusiones}

Las dietas muestran efectos notables en la reducción de peso corporal, pero los cambios en la composición corporal no han sido satisfactoriamente reportados. Los ensayos clínicos no han tenido como objetivo principal la medición de variables de composición corporal. Entre las dos categorías de dietas populares aquí reportadas (de moda y de cambio en el estilo de vida) hay algunas diferencias en la pérdida de peso. La dieta Atkins y de la Zona podrían inducir una pérdida de peso rápida a los 6 meses hasta de $10 \mathrm{~kg}$. Sin embargo, la recuperación del peso perdido a los 12 meses es más probable, pudiendo ser atribuida en gran parte a la baja adherencia. Dietas como el ayuno intermitente que es más flexible promoverían una pérdida de peso a los 6 meses de $5.7 \mathrm{~kg}$ con un tercio de la perdida atribuida a la grasa corporal. En cuanto a la dieta paleolítica, la pérdida de peso que se podría esperar es hasta $8 \mathrm{~kg}$ en seis meses; ocasionada por su poder sacietogénico, similar al que se presenta en las primeras semanas en las dietas cetogénicas. Las dietas Vegetariana y Vegana muestran que la pérdida de peso a los 3 meses es entre 4.3 y $6.5 \mathrm{~kg}$ y se estabiliza a los 6 meses. Los resultados de esta revisión muestran un panorama del efecto de las dietas en la pérdida de peso, pero se sugiere precaución al tomarlos como concluyentes o generalizables. Es difícil la interpretación del efecto de una dieta cuando los estudios incluyen modificaciones en las mismas dietas o adicionan actividad física. En otros casos, como en el ayuno intermitente, la variabilidad de los regímenes no permite extrapolar los mismos resultados a cualquiera de sus variantes.

Así mismo, la falta de datos acerca de la dieta Dukan es un reflejo de las complicaciones y oportunidades para estudiar los efectos en la composición corporal en las dietas populares. Los mismos metanálisis señalan limitaciones para poder explicar los cambios en la grasa subcutánea abdominal o visceral cuando se observa una reducción en el perímetro de cintura. No obstante, comparar los efectos en la composición corporal por efecto de las dietas no es tan confiable como lo es comparar el peso corporal. Es deseable emplear técnicas como la DXA porque la bioimpedancia eléctrica tiene como inconveniente la variación de resultados entre modelos y fabricantes, aunque pueda detectar cambios en el tiempo. Otros detalles que impiden utilizar de mejor manera la información de los ensayos y metanálisis es que no reportan los cambios en el porcentaje de pérdida de peso inicial. Esto permitiría comparar los resultados con las recomendaciones que sugieren un meta inicial de pérdida de peso de $5 \%$ a $10 \%$. Otros índices útiles como el porcentaje de pérdida del exceso de peso son omitidos. Un metanálisis reporto el cambio en el porcentaje de grasa cuando esta métrica es la menos fiable para interpretar cambios en la masa grasa y magra. Finalmente, es necesario remarcar que sigue siendo un pendiente el estudio de los efectos de las dietas después de 12 meses. Aunque no fue el objetivo de esta revisión demostrar cual es la mejor dieta o las más adecuada para perder peso, es necesario señalar lo siguiente: después de evaluar los posibles riesgos a la salud de cualquier dieta, lo siguiente es reconocer que debido a que las distintas dietas son toleradas de manera variable por los individuos, la dieta ideal sería aquella a la que mejor se adhiere una persona y le permite permanecer el mayor tiempo posible con el sostenimiento de los cambios adquiridos. 
Tabla 3. Tipos de dietas basadas en la selección de alimentos por modificación en el estilo de vida o

\section{DIETA PALEOLÍTICA}

El concepto de la dieta paleolítica se originó en década de 1970. Su popularidad se disparó después de la publicación del libro The Paleo Diet: Perder peso y mantenerse saludable al comer los alimentos que fueron diseñados para comer de Loren Cordain en 2002 (Challa, Bandlamud \& Uppaluri, 2020). El principio de la diet es que el cuerpo humano es genéticamente incompatióle con la alimentación modema que surgio a partir silvestres que nuestros ancestros consumian hace más de 10,000 años. La dieta entonces consistia en 55 $65 \%$ (proteína y grasa de origen animal) y $35-45 \%$ (frutas y vegetales). Desatacaban el consumo de pescado, carnes alimentadas de libre pastoreo, verduras, frutas, raices, especias y nueces. Por esta razón, se rechaza la dieta moderna evitando alimentos refinados y ultraprocesados. A pesar de ser una dieta sin restricción calórica o en cantidad de alimentos, resulta saciante por el tipo de alimentos que la conforman (Opie,2014). Al eliminar varios alimentos modernos como granos enteros, legumbres y productos lácteos se considera que

DIETA VEGANA

El término "vegano" fue acuñado por Donald Watson en el primer número de Vegan News, en 1944. Hasta El término "vegano" fue acunado por Don nal Wastorianismo, cuya mayoría de practicantes se dice a a tiliza como un tipo de dieta más, mientras que el veganismo se asume como un estilo de vida basado en la ética (McDonald, 2000). Vegano o vegetariano total, hacen referencia a la exclusión de todos los productos de origen animal como carne, mariscos, aves, huevos y productos lácteos; también elimina derivados de animales como gelatina, miel, albúmina, suero de leche, caseína y algunas formas de vitamina $\mathrm{D}_{3}$ (Thompson, 2008). El EPIC-Oxford mostró que los veganos tenían la ingesta media más baja de vitamina $\mathrm{D}(0,88 \mathrm{\mu g} / \mathrm{d})$. E decir, un cuarto de la ingesta media de los omnivoros. Los veganos tienen que consumir alimentos enriquecidos con vitamina $B_{12} 0$ suplementos que la contengan o podrian presentar signos carenciales. Otros nutrimentos que pueden ser de baja ingesta antre los veganos son los ácidos grasos omega 3 ,

eicosapentaenoico (EPA) yácido docosahexaenoico (DHA) (Nieto, Martinez \& Ros, 2019).

VEGETARIANA

La Asociación Vegetariana Británica fue la primera en emplear la palabra "vegetariano" en el año de 1842 Te a de origen vegetal con el mantenimiento de la salud. En 1960, Felipe Torres y Besola, plantearon los primeros escritos acerca de nutrición vegetariana y el grupo de alimen
la componen (Cayllante, 2014). La Sociedad Argentina de Nutrición (SAN), clasifica las variantes más conocidas del vegetarianismo como: veganos, ovo-vegetariano, lacto-vegetariano, ovolacto-vegetariano y semi-vegetariano. En E.U 3.3\% de los adultos estadounidenses son vegetarianos o veganos, y de ellos $46 \%$ son veganos (Melina, Craig \& levin, 2016).

La dieta vegetariana es considerada un estilo de vida, adoptada bajo principios de filosofia y moral, como la compasión hacia los animales y el cuidado del miedo ambiente, que involucran al individuo a consumir alimentos vegetales. Los vegetarianos se abstienen de consumir carne como aves, pescado y puerco; los puect obstante, si se planifica bien y se incluye verduras, frutas, granos integrales, legumbres, nueces y semillas podría proporcionar una nutrición adecuada (Melina, Craig \& levin, 2016; Cayllante, 2014). Se recomienda que en la dieta vegetariana se incluyan ingestas diarias de vitamina D de 1,000 a 2,000 UI o incluso más. También se sugiere se suplementen con vitamina B12 o se consuman alimentos fortificados ya que 1 pieza de huevo y 1 taza de leche al día aportarian dos terceras parte de las recomendaciones diarias (RDA). En cuanto al Omega-3, la Federación Española de Sociedades de Nutrición, Alimentación y Dietética (FESNAD) sugiere e consumo de 1-1.5 g/día de acido alfa-linolenico (ALA) para cubrir la ingesta entre 53\% y $80 \%$ de las RDA. El calcio en los vegetanianos se ha observado alrededor de $270 \mathrm{mg} / \mathrm{dra}$ (27\% de la $\mathrm{NDA}$ ). En canto al hierro, se

DieTA DE LA ZONA

Esta dieta fue creada por el Dr. Barry Sears en el año 1995. Se basa en equilibrar una comida para optimizar I respuesta hormonal durante las siguientes cinco horas y por tanto controlar los niveles de inflamación de tu cuerpo. Cada tiempo de comida está formado por tres bloques, en proporción $40 \%, 30 \%$ y $30 \%$, carbohidrat proteinas y lipidos, respectivamente (Dario D, 2020). En este tipo de dieta se destaca el consumo de los acidos grasos omega 3 , la mportancla de la sintesis de la insulina y el control de la glucemia. Consta de 5

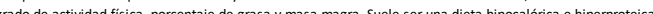
(Dario D, 2020). La postura del Grupo de Revisión. Estudio y Posicionamiento de la Asociación Española de Dietistas-Nutricionistas (GREP-AEDN, 2009) es desaconsejar el método de la dieta de la Zona debido a que, a pesar de contener consejos potencialmente beneficiosos, presenta conceptos que pueden clasificarse como pesar de contener conses
inexactos
Tabla 4. Estudios seleccionados de siete dietas de moda y sus efectos en el peso y composición corporal

\begin{tabular}{|c|c|c|c|}
\hline Autor/año & Dieta & Tiempo & $\begin{array}{l}\text { Efectos en el peso y la } \\
\text { composición corporal }\end{array}$ \\
\hline Johnston et al; 2014 & $\left|\begin{array}{lll}\text { Dieta } & \text { Atkins } & y \\
\text { Dieta } & \text { de } & \text { la } \\
\text { Zona } & & \end{array}\right|$ & 6 meses & $\begin{array}{l}\text { Pérdida de peso } 6 \text { meses } \\
\text { Atkins: }-10.14 \mathrm{~kg} \\
\text { Zona: }-8.4 \mathrm{~kg} \\
\text { Diferencia entre Atkins y Zona: } \\
-1.7 \mathrm{~kg} \\
\text { Pérdida de peso } 12 \text { meses } \\
\text { Atkins: }-6.35 \mathrm{~kg} \\
\text { Zona: }-5.93 \mathrm{~kg} \\
\text { Diferencia entre Atkins y Zona: } \\
-0.42 \mathrm{~kg}\end{array}$ \\
\hline $\begin{array}{l}\text { Cho et al;2019 } \\
\text { Harris et al; } 2018\end{array}$ & $\begin{array}{l}\text { Ayuno } \\
\text { intermitente }\end{array}$ & $\begin{array}{l}1 \text { semana } \\
1 \text { mes } \\
2 \text { meses } \\
3 \text { meses } \\
6 \text { meses }\end{array}$ & $\begin{array}{l}\text { Diferencias en una semana } \\
\text { Peso: }-1.7 \mathrm{~kg} \\
\text { IMC: }-0.5 \mathrm{~kg} / \mathrm{m}^{2} \\
\text { Grasa: } 0 \mathrm{~kg} \\
\text { Diferencias en un mes } \\
\text { Peso: }-1.8 \mathrm{~kg} \\
\text { IMC: }-0.55 \mathrm{~kg} / \mathrm{m}^{2} \\
\text { Masa magra: }-0.55 \mathrm{~kg} \\
\text { Grasa: }-0.73 \mathrm{~kg} \\
\text { Diferencia a } 2 \text { meses } \\
\text { Peso: }-3.2 \mathrm{~kg} \\
\text { IMC: }-3.2 \mathrm{~kg} / \mathrm{m}^{2} \\
\text { Masa magra: }-1.3 \text { a }-1.7 \mathrm{~kg} \\
\text { Grasa: }-2 \mathrm{~kg} \\
\text { Diferencias a } 3 \text { meses } \\
\text { Peso: }-3.4 \mathrm{~kg} \text { a }-5.2 \mathrm{~kg} \\
\text { IMC: }-0.97 \mathrm{~kg} / \mathrm{m} 2 \\
\text { Masa magra: }-0.72 \mathrm{~kg} \\
\text { Grasa: }-2.1 \mathrm{~kg} \text { a }-3.3 \mathrm{~kg} \\
\text { Diferencias a } 6 \text { meses } \\
\text { Peso: }-5.7 \mathrm{~kg} \\
\text { Masa magra: }-1.2 \mathrm{~kg} \\
\text { Grasa: }-3.6 \mathrm{~kg}\end{array}$ \\
\hline $\begin{array}{l}\text { Ghaedi et al;2019 } \\
\text { de Menezes et al; } \\
2019\end{array}$ & \begin{tabular}{|l} 
Dieta \\
Paleolítica
\end{tabular} & $\begin{array}{l}14 \text { días a } 1 \text { mes } \\
3 \text { meses } \\
6 \text { meses }\end{array}$ & $\begin{array}{l}\text { Diferencias a } 14 \text { días- } 1 \mathrm{mes} \\
\text { Peso: }-1 \mathrm{~kg} \text { a }-3.4 \mathrm{~kg} \\
\% \text { de grasa corporal: }-1.34 \% \\
\text { IMC: }-0.8 \mathrm{~kg} / \mathrm{m}^{2} \text { a }-0.9 \mathrm{~kg} / \mathrm{m}^{2} \\
\text { Diferencias a } 3 \text { meses } \\
\text { Peso: }-2.1 \mathrm{~kg} \text { a }-4.1 \mathrm{~kg} \\
\text { IMC: }-1 \mathrm{~kg} / \mathrm{m}^{2} \\
\text { Diferencias a } 6 \text { meses } \\
\text { Peso: }-4.9 \mathrm{~kg} \text { a }-8.1 \mathrm{~kg} \\
\% \text { de grasa corporal: }-1.90 \% \text { a }-4.3 \\
\%\end{array}$ \\
\hline Huang, 2015 & $\begin{array}{l}\text { Dietas } \\
\text { veganas y } \\
\text { Dieta } \\
\text { vegetariana }\end{array}$ & $\begin{array}{l}1 \text { mes } \\
3 \text { meses } \\
6 \text { meses } \\
3 \text { meses } \\
6 \text { meses }\end{array}$ & $\begin{array}{l}\text { Vegetariana } 1 \text { mes } \\
\text { Peso: }-4.6 \mathrm{~kg} \\
\text { Vegetariana } 3 \text { meses } \\
\text { Peso: }-6.5 \mathrm{~kg} \\
\text { Vegetariana } 6 \text { meses } \\
\text { Peso: }-6.5 \mathrm{~kg} \\
\text { IMC: }-2.2 \mathrm{~kg} / \mathrm{m}^{2} \\
\text { Vegana } 3 \text { meses } \\
\text { Peso: }-4.3 \mathrm{~kg} \\
\text { Vegana a } 6 \text { y } 12 \text { meses } \\
\text { Peso: }-4.8 \mathrm{~kg} \\
\end{array}$ \\
\hline
\end{tabular}




\section{Bibliografía}

A Critique of Low-Carbohydrate Ketogenic Weight Reduction Regimens. (1973). JAMA, 224(10), 1415. doi: 10.1001/jama.1973.03220240055018

American Dietetic Association. (2009). Position of the American Dietetic Association: Vegetarian Diets. Journal of the American Dietetic Association, 109(7), 1266-1282.

Anton, S., Hida, A., Heekin, K., Sowalsky, K., Karabetian, C., \& Mutchie, H. et al. (2017). Effects of Popular Diets without Specific Calorie Targets on Weight Loss Outcomes: Systematic Review of Findings from Clinical Trials. Nutrients, 9(8), 822. doi: 10.3390/nu9080822

Anton, S., Moehl, K., Donahoo, W., Marosi, K., Lee, S., \& Mainous, A. et al. (2018). Flipping the Metabolic Switch: Understanding and Applying the Health Benefits of Fasting. Obesity, 26(2), 254-268. doi: 10.1002/oby.22065

Basulto, J., Manera, M., \& Baladia, E., et al. (2012).

Bretón, I. (2016). Fad diets for obesity treatment: a critical review. Anales de la Real Academia Nacional de Farmacia, 82, 195-205.

Cayllante, J. (2014). Vegetarianismo. Rev. Act. Clin. Med, 42, pp. 2195-2199.

Centurión-Bernal, E., González-Acosta, A., RojasPavón, M., Burgos-Larroza, R., \& MezaMiranda, E. (2018). Conocimiento, prácticas y actitudes alimentarias de vegetarianos en Paraguay. Memorias del Instituto de Investigaciones en Ciencias de la Salud, 16(1), 19-25. doi: 10.18004/mem.iics/18129528/2018.016(01)19-025

Challa HJ, Bandlamudi M, Uppaluri KR. Dieta Paleolítica. (2020). En: StatPearl. Treasure Island (FL): StatPearls. Recuperado: https://www.ncbi.nlm.nih.gov/books/NBK48245 7/

Cho, Y., Hong, N., Kim, KW, Cho, SJ, Lee, M., Lee, YH, Lee, YH, Kang, ES, Cha, BS y Lee, BW (2019). La efectividad del ayuno intermitente para reducir el índice de masa corporal y el metabolismo de la glucosa: una revisión sistemática y un metanálisis. Revista de medicina clínica, $\quad 8 \quad(10), \quad 1645$. https://doi.org/10.3390/jcm8101645

Consenso SEEDO 2007 para la evaluación del sobrepeso y la obesidad y el establecimiento de criterios de intervención terapéutica de Menezes, E.V.A., Sampaio, H.A.d.C., Carioca, A.A.F. et al. Influence of Paleolithic diet on anthropometric markers in chronic diseases: systematic review and meta-analysis. Nutr $J$ 18, 41 (2019). https://doi.org/10.1186/s12937-019-0457-z

Federación Mexicana de Diabetes. (2014). Dietas de moda: ni mágicas ni milagrosas. Recuperado de http://fmdiabetes.org/dietas-de-moda-nimagicas-ni-milagrosas/

Dario D. (2020). Qué es la Dieta de la Zona. Recuperado de: https://www.dietadelazona.com.mx/que-es-ladieta-de-la-zona

Feinman, R. D., Pogozelski, W. K., Astrup, A., Bernstein, R. K., Fine, E. J., Westman, E. C., ... Worm, N. (2015). Dietary carbohydrate restriction as the first approach in diabetes management: Critical review and evidence base. Nutrition, 31(1), 1-13.

Foster, G. (2010). Weight and Metabolic Outcomes After 2 Years on a Low-Carbohydrate Versus Low-Fat Diet. Annals Of Internal Medicine, 153(3), 147. doi: 10.7326/0003-4819-153-3201008030-00005

Gardner, C., Kiazand, A., Alhassan, S., Kim, S., Stafford, R., \& Balise, R. et al. (2007). Incorrect Wording and Data Error in: Comparison of the Atkins, Zone, Ornish, and LEARN Diets for Change in Weight and Related Risk Factors Among Overweight Premenopausal Women: The A TO Z Weight Loss Study: A Randomized Trial. JAMA, 298(2), 178. doi: 10.1001/jama.298.2.178

Ghaedi, E., Mohammadi, M., Mohammadi, H., Ramezani-Jolfaie, N., Malekzadeh, J., Hosseinzadeh, M., \& Salehi-Abargouei, A. (2019). Effects of a Paleolithic Diet on Cardiovascular Disease Risk Factors: A 
Systematic Review and Meta-Analysis of Randomized Controlled Trials. Advances In Nutrition, 10(4), 634-646. doi: 10.1093/advances/nmz007

Hernández Alcántara G, Jiménez Cruz A y Bacardí Gascón M. (2015). Efecto de las dietas bajas en carbohidratos sobre la pérdida de peso y hemoglobina glucosilada en personas con diabetes tipo 2: revisión sistemática. Nutrición Hospitalaria, 32, 1960-1966.

Grupo de Revisión, Estudio y Posicionamiento de la Asociación Española de Dietistas-Nutricionistas. (2009) Revisión del libro "La revolucionaria dieta de la Zona". Recuperado de: http://fedn.es/docs/grep/docs/dietazona.pdf

Hamdy (2011). Joslin Diabetes Center \& Joslin Clinic Clinical Nutrition Guideline for Overweight and Obese Adults with type 2 diabetes, prediabetes or those at high risk for developing type 2 diabetes. Recuperado de: https://www.researchgate.net/publication/31884 9674_JOSLIN_DIABETES_CENTER_JOSLIN _CLINIC_CLINICAL_NUTRITION_GUIDELI NE_FOR_OVERWEIGHT_AND_OBESE_AD ULTS_WITH_TYPE_2_DIABETES_PREDIA BETES_OR_THOSE_AT_HIGH_RISK_FOR DEVELOPING_TYPE_2_DIABETES

Harris, L., Hamilton, S., Azevedo, L., Olajide, J., De Brún, C., \& Waller, G. et al. (2018). Intermittent fasting interventions for treatment of overweight and obesity in adults. JBI Database Of Systematic Reviews And Implementation Reports, 16(2), 507-547. doi: 10.11124/jbisrir-2016-003248

Huang, R., Huang, C., Hu, F., \& Chavarro, J. (2015). Vegetarian Diets and Weight Reduction: A MetaAnalysis of Randomized Controlled Trials. Journal of General Internal Medicine, 31(1), 109-116. doi: 10.1007/s11606-015-3390-7

Johnston, B., Kanters, S., Bandayrel, K., Wu, P., Naji, F., \& Siemieniuk, R. et al. (2014). Comparison of Weight Loss Among Named Diet Programs in Overweight and Obese Adults. JAMA, 312(9), $923 . \quad$ doi: 10.1001/jama.2014.10397
Kverneland, M., Molteberg, E., Iversen, P., Veierod, M., Tauboll, E., Selmer, K., \& Nakken, K. (2018). Effect of modified Atkins diet in adults with drug-resistant focal epilepsy: A randomized clinical trial. Epilepsia, 59(8), 1567-1576. doi: 10.1111/epi.14457

La Nueva dieta Atkins. (2020). Recuperado de: http://la.atkins.com/la-nueva-dieta-atkins/

Mansoor, N., Vinknes, K., Veierod, M., \& Retterstol, K. (2015). Effects of low-carbohydrate dietsv. low-fat diets on body weight and cardiovascular risk factors: a meta-analysis of randomised controlled trials. British Journal Of Nutrition, 115(3), 466-479. doi: $10.1017 / \mathrm{s} 0007114515004699$

Martin Gonzalez, I., Plasencia Concepción, D., \& Gonzalez Perez, T. (2001). Manual de dietoterapia. ECIMED.

McDonald, B. (2000). "Once You Know Something, You Can't Not Know It" An Empirical Look at Becoming Vegan. Society \& Animals, 8(1), 1-23.

Melina, V., Craig, W., \& Levin, S. (2016). Position of the Academy of Nutrition and Dietetics: Vegetarian Diets. Journal of the Academy of Nutrition and Dietetics, 116(12), 1970-1980. doi: 10.1016/j.jand.2016.09.025

Nieto, G., Martínez, A., \& Ros, G. (2019). Estudio exploratorio del vegetarianismo en restauración colectiva. Nutrición Hospitalaria. doi: 10.20960/nh.2314

Norma Oficial Mexicana NOM-043-SSA2-2012: Promoción y educación para la salud en materia alimentaria. Recuperado de: http://www.salud.gob.mx/unidades/cdi/nom/com pi/043ssa205.pdf

Norma Oficial Mexicana, NOM-015-SSA2-1994, "Para la Prevención, Tratamiento y Control de la diabetes mellitus en la atención primaria". Al margen un sello con el Escudo Nacional, que dice: Estados Unidos Mexicanos. Recuperado de: http://www.hgm.salud.gob.mx/descargas/pdf/dir gral/marco_juridico/normas/nom_14.pdf 
Opie L. H. (2014). Lifestyle and diet. Cardiovascular journal of Africa, 25(6), 298-301. https://doi.org/10.5830/CVJA-2014-063

Pérez-Lizaur, A., \& García-Campos, M. (2012). Dietas normales y terapéuticas (7a. ed.). México: McGraw-Hill Interamericana.

Rodoreda, J. (2013). Método de la carga de glucógeno o super-compensación. Recuperado el 9 de septiembre del 2020, De: https://www.elsevier.com/eses/connect/medicina/metodo-de-la-carga-deglucogeno-o-super-compensacion.

Dieta Dukan | Dieta $n^{\circ} 1$ en Francia | Dieta Pierre Dukan | acompañamiento adelgazar Dukan | Sin contar calorías |. (2020). Recuperado de http://www.dietadukan.es/

The paleo diet. Built by science. (2020). Recuperado de: https://thepaleodiet.com/

Thompson, J. M. (2008). Nutrición. Madrid, España: PEARSON, EDUCACIÓN.

Trepanowski, J. F., Canale, R. E., Marshall, K. E., Kabir, M. M., \& Bloomer, R. J. (2011). Impact of caloric and dietary restriction regimens on markers of health and longevity in humans and animals: a summary of available findings. Nutrition Journal, 10(1).

Wilhelmi de Toledo, F., Buchinger, A., Burggrabe, H., Hölz, G., Kuhn, C., Lischka, E., ... Michalsen, A. (2013). Fasting Therapy - an Expert Panel Update of the 2002 Consensus Guidelines. Forschende Komplementärmedizin / Research in Complementary Medicine, 20(6), 434-443.

Wolfram, T. (2019). Staying Away from Fad Diets. Recuperado de: https://www.eatright.org/health/weight-loss/faddiets/staying-away-from-fad-diets 